\title{
Números especiales de Revista de Enfermería Vascular dedicados a la terapia de compresión
}

Editorial

En enero de 2022, Revista de Enfermería Vascular celebra su cuarto cumpleaños, y lo celebra lanzando el primer fascículo de una serie de números especiales que van a estar dedicados, de forma exclusiva, a la disección de algo que constituye la piedra angular en el abordaje de los pacientes que sufren o pueden sufrir problemas venosos: la terapia de compresión.

La terapia de compresión para el tratamiento de la enfermedad venosa crónica y el linfedema es una opción de tratamiento no invasiva y fácilmente disponible que está ampliamente documentada. Sin embargo, hay escasez de guías elaboradas a partir de la evidencia aportada por ensayos clínicos para el manejo de pacientes que usan terapia de compresión (1) y, su uso, por desgracia, sigue sin estar generalizado en la práctica clínica. Además, existen diversos estudios (2) que ponen de relieve la falta de adherencia de los pacientes a esta terapéutica.

Tras más de 15 años de labor continuada como enfermero asistencial en una unidad de Cirugía vascular y, tras 10 años de estrecha colaboración con la $\mathrm{AEEVH}$, he podido constatar que este tema sigue generando muchas dudas entre enfermeras y médicos de todos los ámbitos profesionales y, por ello, estoy convencido de que detrás de la renuencia de algunos profesionales y de la falta de adherencia de muchos pacientes, se esconde, entre otros factores, una falta de conocimientos y de formación específica adecuada.

A falta de Guías de Práctica Clínica, una buena forma de aumentar conocimientos es a través de la lectura de artículos científicos, y lo cierto es que localizar literatura relacionada con la terapia de compresión no es difícil puesto que las principales bases de datos bibliográficas internacionales han acuñado descriptores que facilitan la tarea. El problema puede surgir a la hora de intentar realizar una lectura crítica de los artículos encontrados para valorar su calidad y aplicabilidad, o incluso al enfrentarse con las especificaciones técnicas que aparecen en los documentos facilitadas por la industria. Y es que la comprensión de la compresión no es tarea fácil. Comprender la compresión exige el estudio y asimilación de muchos términos y conceptos anatómicos, fisiológicos, fisiopatológicos, semiológicos, diagnósticos y terapéuticos. Pero, además, para poder elegir con criterio entre todas las alternativas disponibles en el mercado es imprescindible conocer otros conceptos relacionados con las propiedades físicas de los tejidos y de los materiales que se usan para confeccionar las vendas, medias y dispositivos de velcro.

El objetivo principal que nos ha empujado a publicar estos números ha sido intentar aportar un poco de luz en estos y muchos otros aspectos relacionados con la compresión para ayudar a las enfermeras y, por supuesto, a todos aquellos profesionales que, en su día a día, necesitan prescribir, dar recomendaciones o aplicar terapia compresiva a sus pacientes. Y para ello, desde el primer momento, en Revista de Enfermería Vascular tuvimos muy claro que la persona más indicada para afrontar con rigor y garantías de calidad este reto era Carmen Alba Moratilla, una reconocida especialista en heridas que ha estado siempre muy comprometida con la formación de otros profesionales en todo lo relativo a la compresión y que ha sido una de las principales impulsoras de su uso en España. Por todo ello, pero también por su carisma, por su capacidad de liderazgo y por sus dotes de comunicación, se ha convertido en todo un referente a nivel nacional e internacional en este campo. Estamos seguros de que, de su mano, esta serie de números especiales que ahora se inicia resultará del interés de todos nuestros lectores.

\section{Bibliografía}

1. Rabe E, Partsch H, Hafner J, Lattimer C, et all. Indications for medical compression stockings in venous and lymphatic disorders: An evidence-based consensus statement. Phlebology. 2018; 33(3):163-84. doi: 10.1177/ 0268355516689631. Epub 2017 Feb 22. PMID: 28549402 ; PMCID: PMC5846867.

2. Chitambira, F. Patient Perspectives: Explaining Low Rates of Compliance to Compression Therapy. 2019. Wound Practice \& Research: Journal of the Australian Wound Management Association. 2019; 27(4): 168-74. Disponible en: https://search.informit.org/doi/10.3316/informit.889357422504811

José María Rozas Martín

Director de Revista de Enfermería Vascular 